Beobachtungen von kleinen Planeten.

\begin{tabular}{|c|c|c|c|c|c|c|c|c|c|c|}
\hline Planet & \multicolumn{2}{|c|}{1905} & M.Z.Wien & Gr. & \multicolumn{2}{|c|}{$\alpha$ app. } & $\log p .4$ & \multicolumn{2}{|c|}{$\delta$ app. } & $\log p . \Delta$ \\
\hline (1 $5^{8}$ ) Koronis & Jan. & 14 & $11^{\mathrm{h}} \quad 8^{\mathrm{m}} 17^{\mathrm{s}}$ & 12.0 & $5^{h} 45^{r}$ & ${ }^{\mathrm{m}} 27.01$ & 9.026 & $+23^{\circ} 3 I^{\prime}$ & I $7 " 7$ & 0.571 \\
\hline $1904 \mathrm{PK}$ & 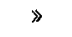 & I 5 & $1034 \quad 46$ & - & 430 & I 5.55 & $9 \cdot 3^{62}$ & +2423 & $2 \cdot 3$ & $0.57^{6}$ \\
\hline $1904 \mathrm{PM}$ & $»$ & 15 & II $55 \quad 39$ & - & 444 & 9.12 & $9.45^{8}$ & +2255 & 46.2 & 0.637 \\
\hline $1905 \mathrm{PR}$ & $\therefore$ & 9 & $1426 \quad 56$ & 13.5 & 546 & $0.62 *)$ & $9 \cdot 56 \mathrm{I}$ & +2236 & $43.5 *)$ & 0.687 \\
\hline 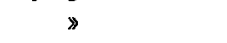 & » & I 4 & 10 5043 & I 3.0 & $5 \quad 42$ & 38.70 & 8.896 & +2224 & I. I & $0.5^{84}$ \\
\hline $9 \circ 5$ PS & ” & I 6 & 1043 & I0.5 & $8 \quad 25$ & 3.69 & $9 \cdot 4^{2} \mathrm{I} n$ & $+\mathrm{I} 849$ & $13 \cdot 5$ & 0.675 \\
\hline $905 \mathrm{PW}$ & » & I 6 & $947 \times 2$ & - & 818 & 58.78 & $9.442_{n}$ & +1716 & 14.8 & 0.695 \\
\hline
\end{tabular}

*) Tritt an Stelle der in A. N. 3989 mitgeteilten genäherten Position.

Photographische Aufnahmen von kleinen Planeten.

\begin{tabular}{|c|c|c|c|c|c|}
\hline Objekt & M.Z.Kgst. & $\alpha$ & $\delta$ & Gr. & $\mathrm{Bb}$. \\
\hline \multicolumn{6}{|c|}{1905 Jan. I 4} \\
\hline $1905 \mathrm{PY}$ & $\left|17^{\mathrm{h}} \mathbf{2}^{\mathrm{m}} \cdot 5\right|$ & $9^{h} \quad 5^{m} \cdot 6$ & $1+15^{\circ} 40^{\circ}$ & - & W \\
\hline
\end{tabular}

1905 Jan. 22.

$1905 \cdot \mathrm{PV}$

$$
\begin{array}{ll|l}
626.6|\mathrm{I} 30.5|+827|13.2| \mathrm{W}
\end{array}
$$

Der Planet ist später von Professor A. Berberich mit (149) Medusa identifiziert worden. Er war auf der Platte die einzige Planetenspur, die einigermaßen zur Position des 6. Jupitermondes vom 4. Januar zu passen schien. Vergleiche hierliber Nr. 3992 p. 143; daselbst sind auch die genauen Ausmessungen der Örter von Jan. 22 und 23 mitgeteilt.

$$
1905 \text { Jan. } 23 .
$$

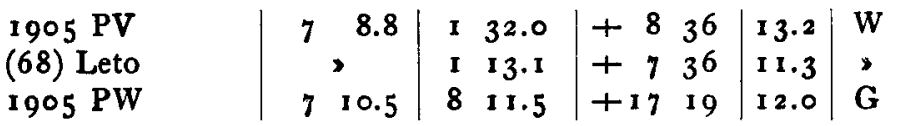

\begin{tabular}{|c|c|c|c|c|c|}
\hline Objekt & M.Z. Kgst. & $\alpha$ & $\delta$ & Gr. & $\mathrm{Bb}$. \\
\hline $\begin{array}{l}\text { 1905 PS } \\
(270) \text { Anahita }\end{array}$ & 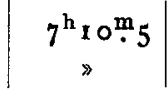 & 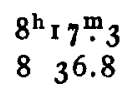 & $\begin{array}{l}+19^{\circ} 0^{\circ} \\
+1528\end{array}$ & $\begin{array}{l}10.2 \\
11.8\end{array}$ & G \\
\hline
\end{tabular}

Astrophys. Institut Königstuhl-Heidelberg, I 905 Jan. 29.
Tägl. Bewegungen: $(68)+I^{m} \cdot 4+I^{\prime}$, PW [bisher irr. tümlich für (5i 7 ) gehaiten] $-\mathrm{Im}^{\mathrm{m}} \circ 0^{\prime}$, PS $-\mathrm{I}^{\mathrm{m}} \mathrm{I}+\mathbf{z}^{\prime}$.

\section{PX \\ (382) Dodona \\ (15) Eunomia \\ 1905 PT \\ $1905 \mathrm{PY}$ \\ (2 I 4) Aschera \\ $1905 \mathrm{PU}$ \\ (5 I) Nemausa}

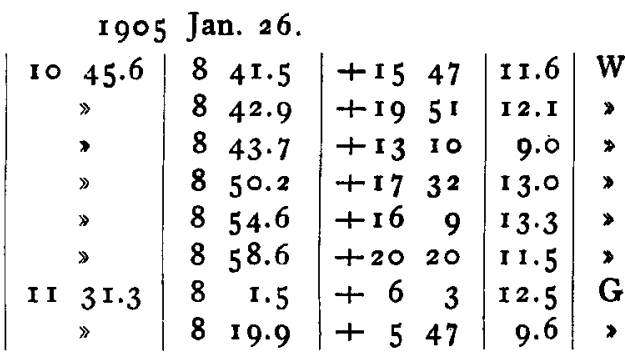

PX und PY sind neue Planeten; PY steht in der Nähe des Oppositionsortes von (427) [1897 DJ], doch ist die Identität nach einer von Prof. H. Kreutz angestellten Untersuchung sehr wenig wahrscheinlich. Tägl. Beweg.: PX $-0^{m} \cdot 9+\mathbf{I}^{\prime}$,

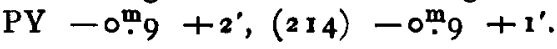

M. Wolf.

\title{
Sur une nouvelle comète à courte période (1904 e).
}

La nouvelle orbite de la comète r $904 \mathrm{e}$, dont on donne plus loin les résultats, met nettement en évidence la courte durée de révolution de cet astre.

Disposant cette fois d'un grand nombre d'observations, on a formé tout d'abord les trois lieux normaux suivants:

$$
\text { t. m. Paris AR. 1905.0 Décl. } 1905.0
$$

I. I 905 Janv. $0.519^{\circ} 4^{\prime} 51^{\prime \prime} .05-8^{\circ} 15^{\prime} 27^{\prime \prime} 3$ I obs. du $3 \circ$ Déc. (Alger); 6 obs. du 3 I Déc. (Königsberg, Copenhague, Marseille, Utrecht, Rome, Alger); 2 obs. du I Janv. (Rome et Copenhague).

$$
\begin{array}{llllllllll}
\text { II. , } & 6.5 & 21 & 9 & 22.40 & -3 & 22 & 5.5 \\
\text { III. }, ~ & 10.5 & 22 & 4 \text { I } & 1.32 & -0 & 9 & 27.4
\end{array}
$$

Comme lieu extrême, on a utilisé une observation du 26 faite à Paris, par M. Bigourdan et qui a donné

$$
\begin{array}{ccc}
\text { t. m. Paris } & \alpha 1905.0 & 81905.0 \\
\text { Janv. 26.26764 } & 29^{\circ} 44^{\prime} 22^{\prime \prime} 65 & +11^{\circ} 48^{\prime} 26 \text {. }^{\prime \prime} 0 .
\end{array}
$$

3 obs. du 5 Janv. (Marseille, et 2 d'Alger); 3 obs. du 7 Janv. (Marseille, et 2 de Besançon).

I obs. du 9 Janv. (Besançon); 4 obs. du ro Janv. (Paris, 2 de Besançon, Vienne); 3 obs. du I I Janv. (Besançon, Paris, Marseille).

Une observation du même jour, faite à Besançon, confirme d'ailleurs ce dernier résultat.

Partant des dates extrêmes, en faisant varier les rayons vecteurs géocentriques $\varrho_{1}$ et $\varrho_{3}$ à ces deux époques, on a d'abord calculé trois orbites qui ont donné les écarts suivants: 
$I^{\theta}$ orbite $2^{\theta}$ orbite $3^{\theta}$ orbite (A) (B)

$$
\begin{aligned}
& \begin{array}{llll}
\log \varrho_{1} & 9.975278 & 9.985829 & 0.015829
\end{array} \\
& \begin{array}{lllll}
\log \varrho_{3} & 0.034199 & 0.045500 & 0.075500
\end{array}
\end{aligned}
$$

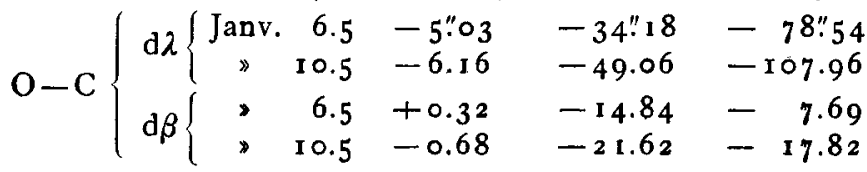

En appellant $x$ et $y$ les facteurs par lesquels il convenait de multiplier les changements (A à B) et (A à $\mathrm{C}$ ), on a ob. tenu les équations de condition qui suivent:

$$
\begin{aligned}
& 2915 x+7341 y=-503 \\
& 4290 x+\text { 10180y }=+616 \\
& \text { 1516x+801 } y=+32 \\
& 2094 x+1714 y=-68
\end{aligned}
$$

qui, traitées par la méthode des moindres carrés, ont fourni, pour valeurs les plus probables de $x$ et $y$

$$
x=+0.04820 \quad y=-0.08332 \text {; }
$$

cela laissait subsister, dans nos équations, des écarts respectivement égaux à: +o.22, - $0.25,-0.26$, +o.26; on ne pouvait guère espérer mieux.

On a ainsi été conduit à entreprendre une dernière recherche avec

$$
\log \varphi_{1}=9.972407 \quad \log \varphi_{3}=0.0313 \circ 3 .
$$

Observatoire de Paris, I 905 Janv. 30.
Il en est résulté le système suivant:

$$
\begin{aligned}
& T=\text { I } 905 \text { Janv, } 16.65370 \text { t. m. Paris } \\
& \omega=35^{\circ} 13^{\prime} 5^{\prime \prime} .98 \\
& \Omega=764 \mathrm{I} 34.49\} 1905.0 \\
& i=303158.75 \\
& \log q=0.145175 \\
& \log e=9.792206 \\
& \mu=503.932 \\
& \log a=0.565090
\end{aligned}
$$

qui paraît très satisfaisant, pour qu'il ne laisse plus subsister,

\begin{tabular}{|c|c|c|c|c|c|c|c|c|c|c|c|}
\hline 1905 & $\alpha$ vera & $\delta$ vera & $\log r$ & $\log \Delta$ & $\mathrm{H}$. & 1905 & $\alpha$ vera & $\delta$ vera & $\log r$ & $\log \Delta$ & H. \\
\hline Febr. 17 & $2^{\mathrm{h}} 49^{\mathrm{m}} 29^{\mathrm{s}}$ & $+25^{\circ} 5^{6: 1}$ & 0.1594 & $0.098 I$ & 0.53 & März 9 & $3^{\mathrm{h}} 45^{\mathrm{m}} 49^{\mathrm{s}}$ & $+35^{\circ} 25^{\prime} \mathrm{I}$ & 0.1805 & 0.16 I I & $0.3^{6}$ \\
\hline I9 & $\begin{array}{rr}5^{2} & 3 \\
54 & 39\end{array}$ & $27 \quad 1.6$ & & & & $1 \mathrm{I}$ & $\begin{array}{rr}40 & 34 \\
52 & 0\end{array}$ & $\begin{array}{ll}35 & 40.5 \\
36 & 11.5\end{array}$ & & & \\
\hline 20 & 57 I 6 & 2733.6 & & & & 12 & $\begin{array}{ll}55 & 7\end{array}$ & $3^{6} \quad 33.9$ & & & \\
\hline 2 I & $2 \quad 59 \quad 55$ & $28 \quad 5.2$ & 0.1630 & 0.1107 & 0.50 & 13 & $358 \quad 16$ & $3^{6} \quad 55.9$ & 0.1855 & $0.173^{6}$ & 0.33 \\
\hline 22 & 3236 & $28 \quad 36.3$ & & & & I 4 & $4 \quad 1 \quad 26$ & $37 \quad 17.4$ & & & \\
\hline 23 & 5 I 8 & 296.9 & & & & I 5 & 438 & $37 \quad 38.4$ & & & \\
\hline 24 & 82 & 2937.0 & & & & I 6 & $75 \mathrm{I}$ & $37 \quad 58.9$ & & & \\
\hline 25 & 1047 & 30 6.7 & 0.1669 & 0.1233 & 0.46 & 17 & I 6 & $3^{8} \quad 18.9$ & 0.1907 & 0.1860 & 0.31 \\
\hline 26 & 1334 & $30 \quad 35.9$ & & & & 18 & 1422 & $\begin{array}{ll}38 & 38.4\end{array}$ & & & \\
\hline 27 & 1622 & $31 \quad 4.6$ & & & & I 9 & 1739 & $3^{8} \quad 57.5$ & & & \\
\hline 28 & I 9 I 2 & 3 I 32.8 & & & & 20 & $20 \quad 57$ & 39 I 6.1 & & & \\
\hline März I & 223 & $\begin{array}{ll}32 & 0.5\end{array}$ & 0.1711 & 0.1359 & 0.43 & 2 I & 24 I6 & 3934.2 & 0.1962 & 0.1983 & 0.28 \\
\hline 2 & $245^{6}$ & $32 \quad 27 \cdot 7$ & & & & 22 & 2737 & 3951.8 & & & \\
\hline 3 & 2750 & $32 \quad 54.5$ & & & & 23 & $3^{\circ} 5^{8}$ & $40 \quad 9.0$ & & & \\
\hline 4 & 3046 & 3320.8 & & & & 24 & $342 I$ & $40 \quad 25.6$ & & & \\
\hline 5 & 3344 & 3346.6 & 0.1757 & 0.1486 & 0.39 & 25 & 3745 & $40 \quad 41.8$ & 0.2018 & 0.2104 & 0.26 \\
\hline 6 & $3^{6} 43$ & 3411.9 & & & & 26 & $4 I 10$ & $40 \quad 57.5$ & & & \\
\hline 7 & 3944 & $34 \quad 3^{6.8}$ & & & & 27 & $443^{6}$ & $41 \quad 12.7$ & & & \\
\hline 8 & $42 \quad 46$ & $35 \quad I .2$ & & & & 28 & $4^{8} \quad 3$ & $41 \quad 27.4$ & & & \\
\hline 9 & $\begin{array}{lll}3 & 45 & 49\end{array}$ & $+3525 \cdot 1$ & 0.1805 & 0.16 I I & 0.36 & 29 & $45^{I} \quad 3 I$ & +4141.6 & 0.2075 & 0.2224 & 0.24 \\
\hline
\end{tabular}
dans les 2 lieux intermédiaires, que les écarts suivants, complètement négligeables dans un calcul à six décimales :

$$
O-C\left\{\begin{array}{ccc}
\text { I } 905 & \mathrm{~d} \lambda & \mathrm{d} \beta \\
\text { Janv. } 6.5 & -0.16 & +0.22 \\
10.5 & -0.58 & -0.24
\end{array}\right.
$$

Il semble donc hors de doute que l'on se trouve en présence d'un astre très intéressant, puisque la période paraît être de 7 ans environ.

On ne peut pas encore décider d'une façon définitive s'il y a identité entre cette comète et celles déjà connues; mais cela paraît improbable, car la comète 1783 , avec laquelle elle présente le plus d'analogie, a des éléments assez différents. Il y aura grand intérêt à suivre cet astre le plus longtemps possible, afin d'être fixé à ce sujet.

\section{Ephemeride des Kometen 1904 e.}

Die folgende Ephemeride ist mit den oben stehenden verbesserten elliptischen Elementen von Fayet für $\mathbf{I}^{\mathrm{h}}$ M. Z. Berlin gerechnet. Einheit der Helligkeit 1904 Dezember 3 ז.5.

Zur Sicherung der Umlaufszeit ist eine möglichst lange Verfolgung des Kometen dringend erwünscht.

Kiel, Bureau der Astron. Nachrichten, 1905 Febr. 2.

E. Strömgren. 